\title{
ON A COVERING PROPERTY OF CONVEX SETS
}

\author{
H. GROEMER ${ }^{1}$
}

\begin{abstract}
Let $\left\{K_{1}, K_{2}, \ldots\right\}$ be a class of compact convex subsets of euclidean $n$-space with the property that the set of their diameters is bounded. It is shown that the sets $K_{i}$ can be rearranged by the application of rigid motions so as to cover the total space if and only if the sum of the volumes of all the sets $K_{i}$ is infinite. Also, some statements regarding the densities of such coverings are proved.
\end{abstract}

If $\left\{K_{i}\right\}=\left\{K_{1}, K_{2}, \ldots\right\}$ is a class of compact convex sets of euclidean $n$-dimensional space $R^{n}$, we say that $\left\{K_{i}\right\}$ can cover $R^{n}$, or that $\left\{K_{i}\right\}$ permits a space covering, if there are rigid motions $\sigma_{i}$ so that $R^{n} \subset \cup_{i=1}^{\infty} \sigma_{i} K_{i}$. The volume of $K_{i}$ will be denoted by $v\left(K_{i}\right)$, and the diameter by $d\left(K_{i}\right)$. In a recent report [1], G. D. Chakerian has discussed the problem of finding necessary and sufficient conditions on $v\left(K_{i}\right)$ and $d\left(K_{i}\right)$ in order that a class $\left\{K_{i}\right\}$ permits a space covering. In the joint paper [2] we have proved, using an idea of G. T. Sallee, the special case $n=2$ of the following theorem:

THEOREM 1. Let $\left\{K_{i}\right\}(i=1,2, \ldots)$ be a class of compact convex subsets of $R^{n}$ with the property that for some constant $M$ and for $i=1,2, \ldots$,

$$
d\left(K_{i}\right) \leqslant M
$$

Then $\left\{K_{i}\right\}$ can cover $R^{n}$ if and only if

$$
\sum_{i=1}^{\infty} v\left(K_{i}\right)=\infty
$$

In the present paper this theorem will be proved in full generality. First we formulate and prove a theorem regarding coverings of the unit cube by a finite number of intervals (Theorem 2). From this theorem it will not only be possible to deduce Theorem 1, but also a more precise statement involving the densities of such coverings (Corollary 1). It should be mentioned that there is a kind of a "dual" of Theorem 2 concerning packings of convex sets rather than coverings (see Kosiński [5]). Theorem 3 shows the possibility of space coverings of density 1 by rather general collections of intervals. As a consequence of this theorem we can deduce another statement (Corollary 2) that establishes, under stronger assumptions than those in Corollary 1 , a smaller bound for the densities of coverings of $R^{n}$ by convex sets.

Received by the editors September 27, 1975 and, in revised form, January 5, 1976.

AMS (MOS) subject classifications (1970). Primary 52A45; Secondary 05B40, 52A20.

Key words and phrases. Convex set, covering, density.

${ }^{1}$ Supported by National Science Foundation Research Grants MPS-03473 A03 and MCS 76-06111. 
The following preliminary remarks are useful in dealing with this class of problems. We define an interval as a subset of $R^{n}$ of the form $\left\{\left(x_{1}\right.\right.$, $\left.\left.x_{2}, \ldots, x_{n}\right): a_{i} \leqslant x_{i} \leqslant b_{i}\right\}$. It can be shown (see Hadwiger [4] or Chakerian [1]) that for every compact convex set $K$ there exists an interval $I$ with

$$
v(I) \geqslant(1 / n)^{n} v(K)
$$

and so that $K$ contains a congruent copy of $I$. Consequently, if $\left\{K_{i}\right\}$ is a class of compact convex sets satisfying (1) and (2) the class $\left\{I_{i}\right\}$ of intervals which can be associated in this way has the same property. It follows immediately that it suffices to prove Theorem 1 only for intervals. Note that the necessity of condition (2) is completely trivial. There is no loss in generality by assuming that $M=1$ in condition (1), since this can always be achieved by an obvious similarity transformation. Furthermore, if $W$ denotes the unit cube

$$
W=\left\{\left(x_{1}, x_{2}, \ldots, x_{n}\right):-\frac{1}{2} \leqslant x_{i} \leqslant \frac{1}{2}\right\}
$$

in $R^{n}$ and if we wish to prove that a class $\left\{I_{i}\right\}$ of intervals satisfying (1) and (2) can cover $R^{n}$, it is sufficient to show that $W$ can be covered by finitely many of these intervals. Indeed, if this is possible one can write $R^{n}$ as a union of unit cubes and cover successively one cube after the other.

These remarks show that Theorem 1 is an immediate consequence of the following theorem.

THEOREM 2. If $I_{1}, I_{2}, \ldots, I_{m}$ are intervals in $R^{n}$ with $d\left(I_{i}\right) \leqslant 1$, and if

$$
\sum_{i=1}^{m} v\left(I_{i}\right) \geqslant 2 \cdot 3^{n-1}-1,
$$

then $W$ can be covered by these intervals using only rigid motions that transform intervals into intervals.

Proof. For $n=1$ the theorem is obvious. We make the induction assumption that it is also true for $R^{n-1}$. The $(n-1)$-dimensional unit cube $W \cap$ $\left\{\left(x_{1}, x_{2}, \ldots, x_{n}\right): x_{n}=0\right\}$ will be denoted by $W^{\prime}$, and the volume in $R^{n-1}$ by $v^{\prime}$. As a further notational convenience we set $c_{n}=2 \cdot 3^{n-1}-1$. For $i=1$, $2, \ldots, m$, let $h_{i}$ be the maximum length of the edges of $I_{i}$, and let $B_{i}$ be a corresponding base of $I_{i}$, that means an $(n-1)$-dimensional face of $I_{i}$ which is orthogonal to an edge of $I_{i}$ of length $h_{i}$. If there are several possibilities qualifying as $B_{i}$ we assume that some selection has been made and is kept fixed. We shall also assume that every $B_{i}$ is contained in the same plane as $W^{\prime}$, i.e. in $x_{n}=0$. This can always be achieved by the performance of a rigid motion of $I_{i}$.

It is obvious that for every $i$,

$$
h_{i} \leqslant d\left(I_{i}\right) \leqslant 1
$$

and

$$
v\left(I_{i}\right)=h_{i} v^{\prime}\left(B_{i}\right) \leqslant v^{\prime}\left(B_{i}\right) .
$$


Furthermore, there is no loss in generality by assuming that

$$
h_{1} \geqslant h_{2} \geqslant \cdots \geqslant h_{m} \text {. }
$$

We define now inductively a finite sequence $k_{0}, k_{1}, \ldots, k_{s}$ of integers by the conditions that $k_{0}=0$ and that $k_{j+1}$ is the smallest integer with

$$
\sum_{i=k_{j}+1}^{k_{j+1}} v^{\prime}\left(B_{i}\right) \geqslant c_{n-1} \text {. }
$$

It follows that $0=k_{0}<k_{1}<\cdots<k_{s} \leqslant m$, where it is always assumed that $s$ is as large as possible. This assumption can be expressed by

$$
\sum_{i=k_{s}+1}^{m} v^{\prime}\left(B_{i}\right)<c_{n-1}
$$

which implies (because of (6))

$$
\sum_{i=k_{s}+1}^{m} v\left(I_{i}\right)<c_{n-1}
$$

It is also of importance to note that we are always concerned with the case $s \geqslant 1$ since (4) and (6) imply that

$$
\sum_{i=1}^{m} v^{\prime}\left(B_{i}\right) \geqslant \sum_{i=1}^{m} v\left(I_{i}\right) \geqslant c_{n}>c_{n-1} .
$$

From the fact that $k_{j+1}$ is the smallest number so that (8) holds it follows that

$$
\sum_{i=k_{j}+1}^{k_{j+1}-1} v^{\prime}\left(B_{i}\right)<c_{n-1},
$$

and since (5) implies $v^{\prime}\left(B_{i}\right) \leqslant d\left(B_{i}\right)^{n-1} \leqslant d\left(I_{i}\right)^{n-1} \leqslant 1$, we can infer that

$$
\sum_{i=k_{j}+1}^{k_{j+1}} v^{\prime}\left(B_{i}\right)<c_{n-1}+1
$$

From (8) and the induction assumption it follows that for $j=0,1, \ldots, s$ -1 the cube $W^{\prime}$ can be covered by each of the collections $\left\{B_{k_{j}+1}\right.$, $\left.B_{k_{j}+2}, \ldots, B_{k_{j+1}}\right\}$. We may assume that these intervals are already arranged so that

$$
W^{\prime} \subset B_{k_{j}+1} \cup B_{k_{j}+2} \cup \cdots \cup B_{k_{j+1}} .
$$

From (6), (7) and (10) we obtain now for $j=0,1, \ldots, s-1$,

$$
h_{k_{j}+1}\left(c_{n-1}+1\right) \geqslant \sum_{i=k_{j}+1}^{k_{j+1}} h_{k_{j}+1} v^{\prime}\left(B_{i}\right) \geqslant \sum_{i=k_{j}+1}^{k_{j+1}} h_{i} v^{\prime}\left(B_{i}\right)=\sum_{i=k_{j}+1}^{k_{j+1}} v\left(I_{i}\right)
$$

and therefore

$$
\begin{aligned}
\sum_{j=0}^{s-1} h_{k_{j}+1}\left(c_{n-1}+1\right) & \geqslant \sum_{j=0}^{s-1} \sum_{i=k_{j}+1}^{k_{j+1}} v\left(I_{i}\right)=\sum_{i=1}^{k_{s}} v\left(I_{i}\right) \\
& =\sum_{i=1}^{m} v\left(I_{i}\right)-\sum_{i=k_{s}+1}^{m} v\left(I_{i}\right) .
\end{aligned}
$$


Because of (4) and (9) we can therefore deduce that

$$
\sum_{j=0}^{s-1} h_{k_{j}+1}>\frac{c_{n}-c_{n-1}}{c_{n-1}+1}=2 .
$$

As an immediate consequence of this inequality and (5) we find

$$
\sum_{j=1}^{s-1} h_{k_{j}+1}>2-h_{k_{0}+1} \geqslant 1 .
$$

Since (7) shows that $h_{k_{j}+1} \leqslant h_{k_{j}}$ we obtain therefore

$$
\sum_{j=1}^{s-1} h_{k_{j}} \geqslant \sum_{j=1}^{s-1} h_{k_{j}+1}>1
$$

If in this inequality the term $h_{k_{s}}$ is added to the left-hand side and $j$ is replaced by $j+1$, it follows that

$$
\sum_{j=0}^{s-1} h_{k_{j+1}}>1
$$

Because of (11) and (7), each union of the form $I_{k_{j}+1} \cup I_{k_{j}+2} \cup \cdots \cup I_{k_{j+1}}$ $(j=0,1, \ldots, s-1)$ contains an interval $J_{j}$ with $W^{\prime}$ as one face and an edge orthogonal to $W^{\prime}$ and of length $h_{k_{j+1}}$. Because of (12) it is clearly possible to translate the intervals $J_{j}$ in the $x_{n}$-direction so that they cover $W$. This finishes the proof.

If $I_{1}, I_{2}, \ldots$ is an infinite sequence with $d\left(I_{i}\right) \leqslant 1$ and $\sum_{i=1}^{\infty} v\left(I_{i}\right)=\infty$, we have $v\left(I_{i}\right) \leqslant 1$, and it follows immediately from Theorem 2 that there is an integer $m$ so that $I_{i}, I_{2}, \ldots, I_{m}$ cover $W$, but $\sum_{i=1}^{m} v\left(I_{i}\right) \leqslant 2 \cdot 3^{n-1}$. The following corollary is an obvious consequence of this latter inequality, together with (3) and the fact that a similarity transformation does not change the density of a covering (see also [3] for the definition of the covering density and the general properties used here).

Corollary 1. Let $\left\{K_{i}\right\}(i=1,2, \ldots)$ be a class of compact convex sets of $R^{n}$ with the property that $\sum_{i=1}^{\infty} v\left(K_{i}\right)=\infty$ and that the set of the diameters $d\left(K_{i}\right)$ is bounded. Then, $\left\{K_{i}\right\}$ can cover $R^{n}$ with a density not greater than $2 \cdot 3^{n-1} n^{n}$. If all the sets $K_{i}$ are intervals this upper bound for the density can be replaced by $2 \cdot 3^{n-1}$.

The bound $2 \cdot 3^{n-1}$ for the densities of coverings by intervals is probably far from the best possible value. In fact, it appears to be unknown whether there exists a sequence of uniformly bounded intervals which permits only space coverings of density greater than 1 . If the volumes of the intervals are bounded away from 0 one can even prove the following theorem.

THEOREM 3. Let $\left\{I_{i}\right\}(i=1,2, \ldots)$ be a class of intervals in $R^{n}$ with the property that there exist constants $M$ and $c$ such that for $i=1,2, \ldots$,

$$
d\left(I_{i}\right) \leqslant M
$$

and 


$$
0<c \leqslant v\left(I_{i}\right) .
$$

Then $\left\{I_{i}\right\}$ can cover $R^{n}$ with density 1 .

Proof. Let $s_{i}^{1}, s_{i}^{2}, \ldots, s_{i}^{n}$ denote the lengths of the edges of the interval $I_{i}$ (in some arbitrary but fixed order). Since it follows from (13) that for every $i$ and $k$,

$$
s_{i}^{k} \leqslant d\left(I_{i}\right) \leqslant M,
$$

and from (13) and (14) that

$$
c \leqslant v\left(I_{i}\right) \leqslant s_{i}^{k} d\left(I_{i}\right)^{n-1} \leqslant s_{i}^{k} M^{n-1},
$$

we obtain

$$
0<c / M^{n-1} \leqslant s_{i}^{k} \leqslant M .
$$

Therefore, if we associate with each interval $I_{i}$ the point $s_{i}=\left(s_{i}^{1}, s_{i}^{2}, \ldots, s_{i}^{n}\right)$ of $R^{n}$, it follows that these points belong to the compact subset $T=\left\{\left(x^{1}\right.\right.$, $\left.\left.x^{2}, \ldots, x^{n}\right): c / M^{n-1} \leqslant x^{k} \leqslant M\right\}$ of $R^{n}$. Consequently, there exists a subsequence of $s_{i}$ that converges to a limit within $T$. An obvious consequence of this fact can be formulated in the following way: There exists a subsequence $J_{1}, J_{2}, \ldots$ of $I_{1}, I_{2}, \ldots$ with the property that the lengths of the edges, say $t_{j}^{1}$, $t_{j}^{2}, \ldots, t_{j}^{n}$ of $J_{j}$ (taken in the previously assigned order) converge to positive limits. Hence, $\lim _{j \rightarrow \infty} t_{j}^{k}=t^{k}$ for some $t^{k}>0$ and every $k=1,2, \ldots, n$. It is now convenient to assume that $t^{1}=t^{2}=\cdots=t^{n}=1$. This assumption entails no real loss in generality since it can always be justified by the application of a suitable nonsingular affine transformation. Therefore, we can now proceed under the assumption that for $k=1,2, \ldots, n$,

$$
\lim _{j \rightarrow \infty} t_{j}^{k}=1 \text {. }
$$

In order to find translations of the intervals $I_{1}, I_{2}, \ldots$ so that $R^{n}$ is covered with density 1 we construct first a sequence of cubes $W_{1}, W_{2}, \ldots$ so that $R^{n} \subset \cup_{j=1}^{\infty} W_{j}$, int $W_{p} \cap$ int $W_{q}=\varnothing$ (for $p \neq q$ ) and that the edge lengths, say $w_{j}$, of the cubes $W_{j}$ satisfy the conditions

$$
\begin{gathered}
w_{j}<1, \\
\lim _{j \rightarrow \infty} w_{j}=1 .
\end{gathered}
$$

It is not difficult to show that such sequences of cubes exist. For example, one can first subdivide $R^{n}$ into the sets $S_{1}=1 \cdot 2 W, S_{2}=2 \cdot 3 W-1 \cdot 2 W$, $S_{3}=3 \cdot 4 W-2 \cdot 3 W, \ldots$ (where $W$ is again the unit cube, $\alpha W=\{\alpha x$ : $x \in W\}$ and the minus sign denotes the set theoretic difference), and then it is easily established that each set $S_{m}$ can be subdivided into $m+1$ "layers" of cubes (surrounding $(m-1) m W$ ) of edge length $m /(m+1)$. The set of these cubes, ordered arbitrarily as a sequence, can obviously serve as the above sequence $W_{j}$.

Because of (15) and (16) it is possible to find for each $W_{j}$ an interval $J_{i_{j}}$ such that an appropriate translate, let us denote it by $J_{j}^{\prime}$, of $J_{i j}$ covers $W_{j}$. 
Moreover, this selection can clearly be made so that no $J_{i_{j}}$ is used more than once in this process. We have now to show that the sequence $J_{1}^{\prime}$, $J_{2}^{\prime}, \ldots$, which is certainly a covering of $R^{n}$, has density 1 . For this purpose let $B_{r}$ denote a solid sphere in $R^{n}$ centered at the origin and of radius $r$, and let $d_{r}$ be defined by

$$
d_{r}=\frac{1}{v\left(B_{r}\right)} \sum_{J_{j}^{\prime} \subset B_{r}} v\left(J_{j}^{\prime}\right) .
$$

Then the density $\delta$ of the covering $\left\{J_{j}^{\prime}\right\}$ is given by $\delta=\lim _{r \rightarrow \infty} d_{r}$.

It follows from (15) and (17) that for any positive $\varepsilon$ one can find a number $N$ so that for all $j>N, v\left(J_{j}^{\prime}\right) / v\left(W_{j}\right)<1+\varepsilon$. Consequently, if we set $\sum_{j \leqslant N} v\left(J_{j}^{\prime}\right)=Q$ and notice that $J_{j}^{\prime} \subset B_{r}$ implies $W_{j} \subset B_{r}$ we obtain

$$
\begin{aligned}
d_{r} & \leqslant \frac{Q}{v\left(B_{r}\right)}+\frac{1}{v\left(B_{r}\right)} \sum_{J_{j}^{\prime} \subset B_{r}, j>N} v\left(J_{j}^{\prime}\right) \\
& \leqslant \frac{Q}{v\left(B_{r}\right)}+\frac{1}{v\left(B_{r}\right)} \sum_{W_{j} \subset B_{r}, j>N}(1+\varepsilon) v\left(W_{j}\right) \leqslant \frac{Q}{v\left(B_{r}\right)}+(1+\varepsilon) .
\end{aligned}
$$

Since $Q$ does not depend on $r$, we have $\lim _{r \rightarrow \infty} Q / v\left(B_{r}\right)=0$ and, therefore,

$$
\delta=\lim _{r \rightarrow \infty} d_{r} \leqslant 1+\varepsilon
$$

If we note that for any covering $\delta \geqslant 1$ we obtain for $\varepsilon \rightarrow 0$ that $\delta=1$. But at this point the proof of our theorem is not yet completely finished, since only a subclass of the originally given set of intervals has been used for the described covering. However, the intervals which have not been used, let us call them $K_{1}, K_{2}, \ldots$, can be distributed (by the application of translations) so thinly that they have density 0 . For example, if each $K_{i}$ is translated so that it has distance $i^{2}$ from the origin then, using (13) we find

$$
\lim _{r \rightarrow \infty} \frac{1}{v\left(B_{r}\right)} \sum_{K_{i} \subset B_{r}} v\left(K_{i}\right) \leqslant \lim _{r \rightarrow \infty} \frac{1}{v\left(B_{r}\right)} \sum_{i^{2}<r} M^{n} \leqslant \lim _{r \rightarrow \infty} \frac{\sqrt{r}}{v\left(B_{r}\right)} M^{n}=0 .
$$

Since $\left\{J_{j}^{\prime}\right\}$ has density 1 and $\left\{K_{i}\right\}$ has density 0 it follows that $\left\{J_{j}^{\prime}\right\} \cup\left\{K_{i}\right\}$ has density 1 , and the proof of Theorem 3 is finished.

The following corollary is an immediate consequence of (3) and Theorem 3. It is of the same type as Corollary 1 but it provides a better estimate under stronger assumptions.

COROllary 2. Let $\left\{K_{i}\right\}(i=1,2, \ldots)$ be a class of compact convex sets of $R^{n}$ with the property that the set of the diameters $d\left(K_{i}\right)$ is bounded from above, and the set of the volumes $v\left(K_{i}\right)$ is bounded away from 0 . Then $\left\{K_{i}\right\}$ can cover $R^{n}$ with density not greater than $n^{n}$.

Finally, it might be worth mentioning that Theorem 3 can also be formulated for packings instead of coverings and that the packing version can be proved by essentially the same methods as the covering theorem. 


\section{REFERENCES}

1. G. D. Chakerian, Covering space with convex bodies, The Geometry of Metric and Linear Spaces (Michigan, 1974), Lecture Notes in Math., vol. 490, Springer-Verlag, Berlin and New York, 1975, pp. 187-193.

2. G. D. Chakerian and H. Groemer, On classes of convex sets that permit plane coverings, Israel J. Math. 19 (1974), 305-312.

3. H. Groemer, Existenzsätze für Lagerungen im Euklidischen Raum, Math. Z. 81 (1963), 260-278. MR $29 \# 525$.

4. H. Hadwiger, Volumschätzung für die einen Eikörper überdeckenden und unterdeckenden Parallelotope, Elem. Math. 10 (1955), 122-124. MR 17, 998.

5. A. Kosiński, A proof of an Auerbach-Banach-Mazur-Ulam theorem on convex bodies, Colloq. Math. 4 (1957), 216-218. MR 19, 165.

Department of Mathematics, University of Arizona, Tucson, Arizona 85721 